\title{
On the characterisation of the normal population by the independence of the sample mean and the sample variance.
}

Tatsuo Kawata and Heihati Sakamoto.

\author{
(Read May 6, 1944.) \\ (Received July 12, 1944.).
}

1. Let $X_{1}, X_{2}, \ldots \ldots, X_{n}(n \geqq 2)$ be the sample variables from a certain population, that is, let $X_{i}(i=1,2, \ldots \ldots, n)$ be independent random variables having same distribution $F(x)$. In the mathematical statistics, the following fact is well known and is of fundamental importance in the theory of exact 'sampling.

If $F(x)$ is the normal distribution function, then the two statistics

$$
\bar{X}=\frac{1}{n} \sum_{i=1}^{n} X_{i}
$$

and

$$
S=\frac{1}{n} \cdot \sum_{i=1}^{n}\left(X_{i}-\bar{X}\right)^{2}
$$

are statistieally independent.

R. C. Geary ${ }^{(1)}$ has proved the converse of this theorem and given the characterisation of the normal population by using the formulae ${ }^{(2)}$ due to Fisher for relations between semi-invariants of various algebraic forms of sample variables. The object of the present paper is to give another proof, under the more general conditions assuming nothing about the moments of $X_{i}$, while Geary has supposed the existence of moments of every order.

2. We restate the theorem.

Theorem. Let $X_{1}, X_{2}, \ldots \ldots, X_{n}(n \geq 2)$ be the independent, random variables zuhose distributions are equal to the same $F\left(x_{1}\right)$. If two random variables $Y=\sum_{i=1}^{n} X_{i}, Z=\sum_{i=1}^{n}\left(X_{i}-\bar{X}\right)^{2}\left(\bar{X}=\frac{1}{n} \sum^{n} X_{i}\right)$ are independently distributcd,

(1) R. C. Geary, The distribution of "Student's" ratio for non-normal samples. Journ. Royal Statist. Soc., Supplement 3 (1936).

(2) R. A. Fisher, Moments and product moments "of sampling distributions. Proc. London Math. Soc. 30 (1929). 
then $F(x)$.must be the normal distribution function, excluding the wnit distribution.

We consider the characteristic function ${ }^{(3)}$ of the simultaneous variable. $\left(X_{i}, X_{i}^{2}\right)$

$$
f(t, s)=\int_{-\infty}^{\infty} e^{i \ell x+i s x^{2}} d F(x),
$$

where $t$ is a real number but we consider $s$ as a complex number, $s=\sigma+i \tau$, $\tau>0 . f(\dot{t}, s)$ is obvlously an analytic function of $s$ regular in the upper half -plane $\tau>0$. Since $X_{i}(i=1,2, \ldots \ldots, n)$ are independent variables, the characteristic function of variable $(Y, \Sigma), \Sigma$ being $\sum_{i=1}^{n} X_{i}^{o}$, is $\{f(t, s)\}^{n}$ which noticing that $\Sigma \geq 0$, can also be written as

$$
\int_{-\infty}^{\infty} \int_{0}^{\infty} e^{i \ell \eta+i s n} d F(\eta, \theta) \text {, }
$$

where $F(\eta, \theta)$ is the distribution function of $(Y, \Sigma)$.

Since $Z+\frac{1}{n} Y^{2}=\Sigma$, denoting the distribution of $(Y, Z)$ as $G(\eta, \zeta)$, we have further

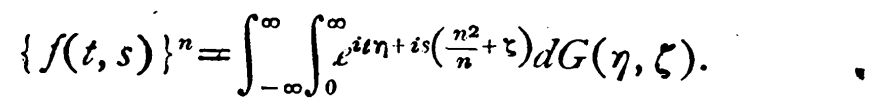

The statistical independence of $Y$ and $Z$ shows that

$$
d G(\eta, \zeta)=d G_{1}(\eta) d G_{2}(\zeta)
$$

$G_{1}(\eta)$ and $G_{2}(\zeta)$ being the distribution function of $Y$ and $Z$ respectively. Hence we can write $(2 \cdot 3)$ as

$$
\{f(t, s)\}^{n}=\int_{-\infty}^{\infty} e^{i \ell \eta+i \frac{s}{n} \eta^{2}} d G_{1}(\eta) \cdot \int_{0}^{\infty} e^{i \varepsilon \zeta} d G_{2}(\zeta) .
$$

Now we observe that, putting $\phi\left(t,-\frac{s}{n}\right)=\int_{-\infty}^{\infty} e^{i\left(\eta+i \frac{s}{n} \eta^{2}\right.} d G_{1}(\eta)$ and $\alpha(s)$ $=\mu_{n}(s)=\int e^{i s \zeta} d G_{2}(\zeta)$,

(3) In the ordinary sense of the characteristic function, ' $t, s$ are real, but in this paper, we use the same terminology in the case where $s$ is complex. 


$$
\begin{aligned}
& -i-\frac{\partial^{2}}{\partial t^{2}} \dot{f}(t, s)=-\frac{\partial}{\partial s} f(t, s) ; \quad \tau>0, \\
& -\frac{i}{n} \frac{\partial^{2}}{\partial t^{2}} \psi\left(t, \frac{s}{n}\right)=\frac{\partial}{\partial s} \varphi\left(t, \frac{s}{n}\right), \quad \tau>0,
\end{aligned}
$$

and

$$
-i \alpha^{\prime}(s) \geqq 0 \text { for } \sigma=0, \tau>0 .
$$

We differentiate both sides of $(2 \cdot 5)$ with respect to $s$, we have

$$
n\{f(t, s)\}^{n-1} \frac{\partial}{\partial s} f(t, s)=\alpha(s)-\frac{\partial}{\partial s} \psi\left(t, \frac{s}{n}\right)+\psi\left(t, \frac{s}{n}\right) \alpha^{\prime}(s) \cdot{ }^{(4)}
$$

which becomes, by $(2 \cdot 6)$ and $(2 \cdot 7)$,

$$
n\{f(t, s)\}^{n-1} \frac{\partial^{2}}{\partial t^{2}} f(t, \dot{s})=\frac{1}{n} u(s) \frac{\partial^{2}}{\partial t^{2}} \psi\left(t, \frac{s}{n}\right)+i \psi\left(t, \frac{s}{n}\right) u^{\prime}(s) .
$$

In differentiating two times $(2 \cdot 5)$ with respect to $t$ we get

$$
\begin{aligned}
& n\{f(t, s)\}^{n-1} \frac{\partial^{2}}{\partial t^{2}} f(t, s)+n(n-1)\{f(t, s)\}^{n-1}\left\{\frac{\partial}{\partial t} f(t, s)\right\}^{2} \\
& =u(s) \frac{\partial^{2}}{\partial t^{2}} \psi\left(t, \frac{s}{n}\right) .
\end{aligned}
$$

The elimination of $u(s) \frac{\partial^{2}}{\partial t^{2}} \psi\left(t, \frac{s}{n}\right)$ from $(2 \cdot 9)$ and $(2 \cdot 10)$ gives

$$
\left.\{f(t, s)\}^{n-1} \frac{\partial^{2}}{\partial t^{2}} f(t, s)-\{f, t, s)\right\}^{n-1}\left\{\frac{\partial}{\partial t} f(t, s)^{2}\right\}=i \frac{a^{\prime}}{n-1} \psi\left(t, \frac{s}{n}\right) .
$$

By $(2 \cdot 5)$, this becomes further

$$
\begin{gathered}
\{f(t, s)\}^{n-1} \frac{\partial^{2}}{\partial t^{2}} f(t, s)-\{f(t, s)\}^{n-2}\left\{\frac{\partial}{\partial t} f(t, s)\right\}^{2} \\
=i\{f(t, s)\}^{n} \frac{1}{n-1} \frac{\alpha^{\prime}(s)}{u(s)}
\end{gathered}
$$

From this equation we can easily prove that in the $t$-interval, for fixed $s$, such that $f(t, s) \neq 0$,

(4) The dash in $a^{\prime}(s)$ means the differentiation with respect to $s_{0}$ 


$$
f(t, s)=\exp \left[\frac{i}{n-1} \frac{\alpha^{\prime}(s)}{\alpha(s)}\left\{\frac{t^{2}}{2}+C(s) t+D(s)\right\}\right] .
$$

But since $f(t, s)$ is a continuous function of $t$ and the right side of $(2 \cdot 12)$ has no zeros as a function of $t$, we see that $(2 \cdot 12)$ holds for all values of $t$.

Now we take $\sigma=0$, and thus $s=i \tau$. Then it holds that

$$
\lim _{\tau \rightarrow+0} f(t, i \tau)=\int_{-\infty}^{\infty} \cdot e^{i \ell x} d F(x)
$$

for every $t$, since

$$
\begin{aligned}
& \left|\int_{-\infty}^{\infty} e^{i \ell x} d F(x)-\int_{-\infty}^{\infty} e^{i \ell x-\tau x^{2}} d F(x)\right| \leqq\left|\int_{-A}^{A} \epsilon^{i \ell \tau}\left(1-e^{-\tau x^{2}}\right) d F(x)\right| \\
+ & \left|\int_{|x|>A} e^{i \ell x} d F(x)\right|+\left|\int_{\mid x !>A} e^{\imath \ell x-\tau x^{2}} d F(x)\right| \leqq \tau A^{2} \int_{-A}^{A} d F(x)+2 \int_{|x|>A} d F(x)<\varepsilon,
\end{aligned}
$$

if we take $A$ such that $2 \int_{|x|>A} d F(x)<\frac{\varepsilon}{2}$ and then take $\tau$ so small that

$$
\tau A^{2} \int_{-A}^{A} d F(x)<\frac{\varepsilon}{2}
$$

Now if we take $t=0$ in $(1 \cdot 12)$ and let $\tau$ tend to zero, then by $(2 \cdot 13)$ $f(0, i \tau) \rightarrow 1$, and hence

$$
\lim _{\tau \rightarrow 0} \frac{\alpha^{\prime}(i \tau)}{u(i \tau)} D(i \tau)=0
$$

Next noticing the existençe of $\lim _{\tau \rightarrow 0} f(t, i \tau) f(-t, i \tau)^{\prime}, \quad(t \neq 0)$, we can show the existence of $\lim _{\tau \rightarrow 0} \alpha^{\prime}(i \tau)$. And hence we also get the existence of $\lim _{\tau \rightarrow 0} C(i \tau)$.

Let

$$
\lim _{\tau \rightarrow 0} \frac{i}{n-1} \frac{\mu^{\prime}(i \tau)}{\alpha(i \tau)}=-u_{n}, \quad \lim _{\tau \rightarrow 0} \frac{i}{n-1} \frac{\alpha^{\prime}(i \tau)}{u(i \tau)} C(i \tau)=\beta_{n} .
$$

If $\alpha_{n} \neq 0$, then letting $s=i \tau \rightarrow 0$ in $(2 \cdot 12)$, we have

$$
f(t)=f(t, 0)=e^{-\frac{\alpha n}{2} t^{2}+\beta_{n} t}
$$

But since the left side is independent of $n, \alpha_{n}$ and $\beta_{n}$ are constants in- 
dependent of $n^{(5)}$ and thus we can put $\alpha_{n}=\alpha, \beta_{n}=i \beta$, where $\beta$ is real, for $f(t)=\overline{f(-t)}$.

If $\alpha \neq 0$; then by $(2 \cdot 8), \alpha>0$ and $(2 \cdot 14)$ can be written as

$$
f(t)=e^{-\frac{\alpha}{2} t^{2}+i \beta t}
$$

which shows that $F(x)$ is a normal distribution function.

If $u=0$, then $f(t)=e^{i 3 t}$. This shows that $F(x)$ is an unit distribution function having only one point spectrum at $x=\beta$.

(5) This can also be proved explicitly. From the existence of $\lim a_{n}$ we can show that the variance of $Z$ is finite, and $\frac{1}{i} \lim _{\tau \rightarrow 0} \alpha^{\prime}(i \tau)=E(Z)$, the mean value of $Z$, which is $(n-1) \sigma^{2}$, $\sigma^{2}$ being the variance of $X_{i}$. This is a well known fact in the sampling theory. Hence $a_{n}$ is independent of $n$. For $\beta_{n}$, we can also prove its independence of $n$ directly. 